\title{
FOIRIER IMAGES QUASISYMMETRY OF GENERALIZED TRIADIC KOCH PREFRACTALS
}

\section{Galina V. Arzamastseva, Mikhail G. Evtikhov, Feodor V. Lisovsky, Ekaterina G. Mansvetova}

Kotel'nikov Institute of Radioengineering and Electronics, Fryazino Branch, Russian Academy of Sciences, http://fire.relarn.ru 1, Vvedensky sq., 141120 Fryazino, Moscow region, Russian Federation arzamastseva@mail.ru, emg20022002@mail.ru, lisf@df.ru,mansvetova_eg@mail.ru

Abstract. The study of the Fourier-images properties was made by numerical methods for the family of flat triadic geometric prefractals with generator in the form of symmetric four-stage broken line with an arbitrary angle at the apex between the central units and the initiator in the form of a straight line (Koch curve) or in the form of an equilateral triangle (the Koch snowflake). To obtain the Fourier images the pictures of fractals were approximated by a grid function on a uniform grid with cells small enough for adequate mapping of high generation prefractal details, and then were digitized in order to use fast Fourier transform for determination the values of the squared modules of the Fourier component, that is, the spectral intensity distribution of diffraction maxima in the Fraunhofer region. An analysis showed that for the values of the vertex or base angles equal to the integer fraction of 180 degrees, Fourier images are the same as for the perfect crystals with the symmetry axes of the 2-nd, 3-th, 4-th and 6-th order, or as for parcuet mosaics or quasicrystals with the axes of quasisymmetry of any order. Really in the Fourier images of the Koch curves with the initiator in the form of a straight line was observed axis of quasisymmetry from 3rd to 9 th and 11-th order. Similar to the above-described properties are also inherent to Fourier images of the Koch snowflake with the initiator in the form of an equilateral triangle. The configuration of the observed Fourier images can be approximately regarded as a radial-annular, at that in the peripheral ("lattice") of the images is dominated by the radial nature of the frequency distribution of diffraction reflections along the radius, and in the central ("fractal") - a ring with self-similarity. The lattice part has a kind of clustering: all the rays have a strong central chain of reflexes along the radii and parallel to it the weaker satellites on both sides. All Fourier images had the center of symmetry, which is an integral attribute of the diffraction patterns in the Fraunhofer zone for any objects, however, the rotational symmetry was not perfect: the positions of the diffraction reflexes when rotating images at angles that correspond to the order of the symmetry axis remain unchanged, but their intensity could vary. The cause of the observed features is that prefractals, unlike crystals, are not a continuum of point objects but two-dimensional set of equal length line segments with different orientation in space. In this set for the considered configurations of the generator it is possible to allocate several two-dimensional subsets with equally oriented segments, each of which contains a number of partial one-dimensional diffraction gratings formed by segments located along the same line. These parallel lattice in the general case contain a different number of segments, and the degree of filling and the distance between adjacent segments, determining the intensity and diffraction reflections distribution structure along the line, depend on the orientation of the lattice and the generation number of prefractal.

Keywords: digital methods, Fourier image, Fraunhofer diffraction, generator, initiator, Koch curve, Koch fractal, Koch snowflake, parquet mosaic, prefractal, quasiqristal, quasisymmetry, scaling invariance, selfsimilarity, symmetry.

UDC 51.74; 535.42

Bibliography -47 references

Received 06.12.2016

RENSIT, 2016, 8(2):207-214

DOI: $10.17725 /$ rensit.2016.08.207

Contents:

1. INTRODUCTION (208)

2. Obtaining a Fourier-images of the Koch CURVES AND SNOWFLAKES (208)

3. Configuration and Symmetry of the

FouriER-IMAGES (209)
4. SCHEME OF THE LATTICE AND FRACTAL PARTS OF THE FOURIER-IMAGES FORMATION (210)

5. Summary (212)

References (212) 


\section{INTRODUCTION}

Fractal ideology, originally considered only as the basis of exotic branches of abstract mathematics, at the present stage is widely used not only to describe and analyse the various material objects and phenomena [1-4], but persistently intrudes into many areas of human activity. Engineering and technology [5, 6], biology and medicine [7], economics $[8,9]$, art (music, painting, literature, linguistics, architecture) [10-14] - this list could easily be continued. However, from the point of view of the science of fractals priority areas continue to be math and physics [15].

At the end of XX century there was a marked interest in the study of Fourier-images of flat fractals (i.e., a diffraction pattern in the Fraunhofer zone) (see eg. [16-18]), which remaines unabated to this day.

In particular, this is due to the fact that by using Fourier images one can fairly easy determine Hausdorff dimension of the studied objects [16]. Authors of papers [17-18] for the first time performed experiments on the scattering of light by microphotographs of the Koch snowflake in order to get the Fourierimage of it and compared obtained data with the theory using the analytical description of a fractal. The features of Fraunhofer diffraction for other fractal objects (Vicsek fractal, Cantor bar, Sierpinski carpet, etc. [19-24]) were also investigated.

The diffraction method of $D_{f}$ determination is widespread, as it is applicable to any objects including real fractals which are not subject to any geometrical or analytical description. However, the utilitarian usefulness of the Fourier transform images is not limited by this, because they can be used to obtain information about the structure and symmetry of abstract fractals, and about phase transitions in real media.

For example, the Fourier images analysis of nonuniform magnetization distributions allowed to detect the phenomenon of biperiodic domain structures amorphization in quasi-uniaxial magnetic films with critical thickness [25], and the presence of phase transitions of "devil's staircase" type in magnetic films with modulated structure [26]. It is not necessary to ignore the aesthetic factor: often talk about the beauty of fractals [27], but the beauty of their Fourier images is just as impressive [28].

\section{OBTAINING A FOURIER-IMAGES OF THE KOCH CURVES AND SNOWFLAKES}

To obtain Fourier images we used numerical algorithm, the essence of which is as follows. Images of studied fractals are approximated by a uniforn grid function with cells small enough for adequate reproduction of the high generations pre-fractals details. For digitized pictures using fast Fourier transform we determined the values of the Fourier component squared modules, i.e. the spectral distribution of intensity $I$ of diffracted radiation in the zone of Fraunhofer. To display the intensity $I$ of the diffraction maxima on the 2D-plane, we use representation of $I$ values in a form of circles with radii proportional to logarithm of $I$, where coefficient of proportionality is chosen for reasons of optimal illustrativity of images. With the same purpose Gaussian blur of displaying circles was additionally applied.

The described above procedure of the Fourier images determining was for the first time described and applied by us [29] to study the fractal-like domain structures in opaque magnetic films (see [30-32]), wfs later set forth in public periodicals [25] and tested on the probe objects (mono- and biperijdical domain structures in transparent magnetic films), where these objects and diffraction patterns created by them could be observed visually in the transmission mode and photographed [25, 26]. Using digitized images were obtained Fourier images for many flat geometric and algebraic fractals: the Sierpinski carpet, $H$-fractals, Peano curves, bifractals, L-system fractals, Mandelbrot sets $[28,32,33-35]$. 
In this article the study of the Fourierimages properties was made by numerical methods for the family of flat triadic geometric prefractals of generation with generator in the form of symmetrical relative to the middle four-stage broken line with an arbitrary angle $0^{\circ}<\alpha<180^{\circ}$ at the apex between the central units (Fig. 1a) and the initiator in the form of a straight line (Koch curve) or in the form of an equilateral triangle (the Koch snowflake); (see Fig. 1b). As an example, in Fig. 1c a view of second generation Koch prefractals is given. To preserve the self-similarity of prefractal by iterations all the segments of generator in a form of broken line must have the same length $L / 3^{N_{g}}$. When $\alpha \rightarrow 0^{\circ}$, the broken line becomes delta-shaped, when $\alpha \rightarrow$ $180^{\circ}$, it merges with a initiator line; case $\alpha=$ $60^{\circ}$ corresponds to the classical Koch curve.

An analysis showed that for $\alpha=180^{\circ} / k_{\alpha}$ or $\gamma=90^{\circ}-\alpha / 2=180^{\circ} / k_{\gamma}$, where $k_{\alpha}$ and $k_{\gamma}-$ any integer numbers, Fourier images are the same as for the perfect crystals with the symmetry axes of the 2-nd, 3-rd, 4-th and 6-th order, or as for parcuet mosaics [36] or quasicrystals [37] with

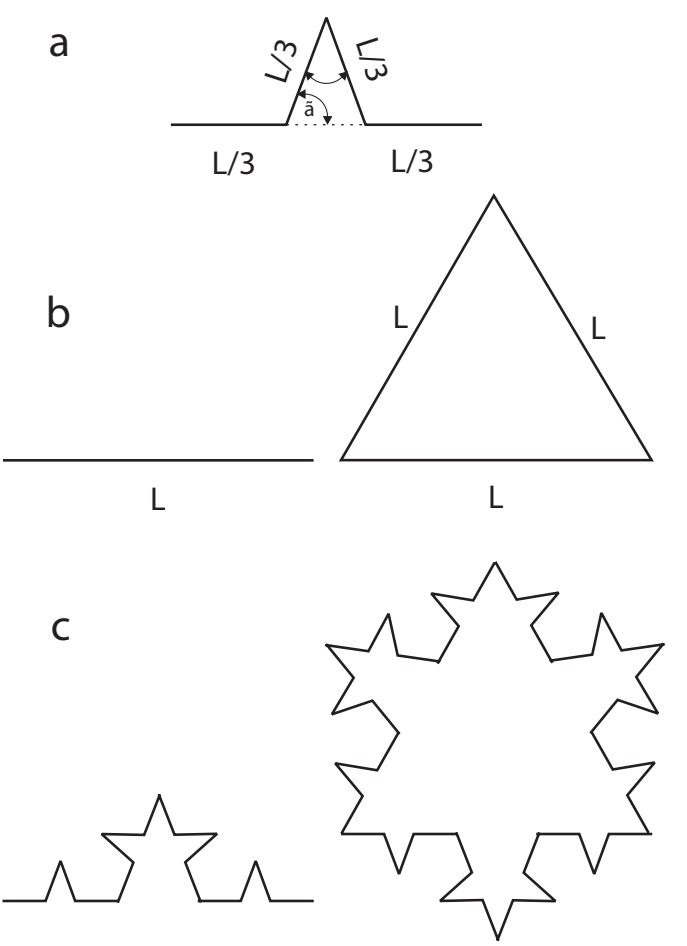

Fig. 1. Generator (a), initiators (b) and prefractals of the 2-nd generation for Koch curve and snowflake (c). the axes of quasisymmetry of any order. Really among the Fourier images of the Koch curves with the initiator in the form of a straight line were observed axis of quasisymmetry from 3-rd to 9 th and 11-th order. High quality Fourier image with the axis of quasisymmetry of 10-th order failed to obtain; the reason for this will be discussed next. For integer values of $k_{\alpha}$ only axes quasisymmetry of odd order exist (3-rd to 11-th inclusive), while for integer values of $k_{\gamma}-$ any axis with the order in the range of 3 to 9. In Fig. 2 Fourier images of 6 -th generations Koch prefractals for $\alpha=36^{\circ}-(a), \alpha=25.743^{\circ}-(b)$, $\alpha=20^{\circ}-(c), \alpha=16.2637^{\circ}-(d)$ are shown.

\section{CONFIGURATION AND SYMMETRY OF THE FOURIER-IMAGES}

The configuration of the observed Fourier images can be approximately regarded as a radial-annular, and, as was first noted by the authors of [17] for a classic example of Koch snowflake, in the peripheral ("lattice") of the images the radial nature of the frequency distribution of diffraction reflects is dominated, and in the central ("fractal") - a ring nature with
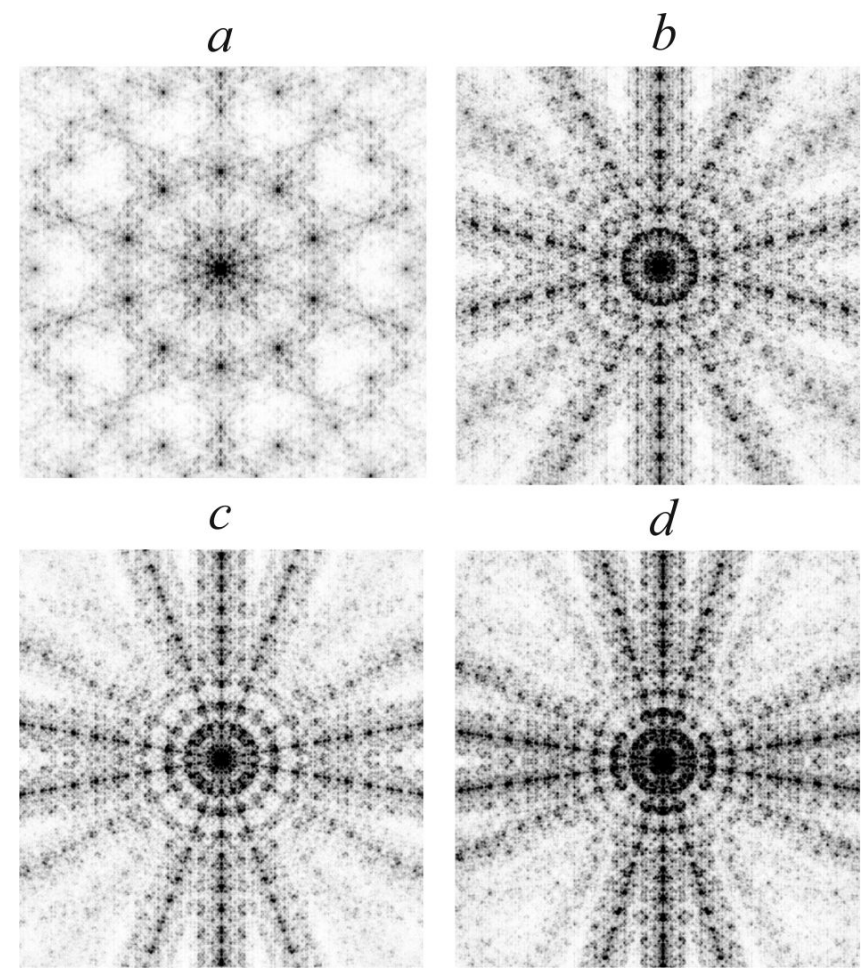

Fig. 2. Fourier images of Koch curve prefractals with quasisymmetry axis of 5-th (a), 7-th (b), 9-th (c) and 11-th (d) order. 
self-similarity. It was found that the diameter of the central region after each iteration increasing in $m$ times, where $m=2[1+\sin (\alpha / 2)]$ is the scale factor for the considered fractals.

The lattice part has a kind of clustering: all the rays have a strong central chain of reflexes along the radii and parallel to it the weaker satellites on both sides (see Fig. 2b-d). Similar features can be traced also in Fig. 3, where Fourier images for prefractal of the Koch curves of 6-th (a), 5-th (b) and 4-th $(c-d)$ generations for the values $k_{\gamma}=$ $3,4,6$ and 8 are shown.

All Fourierimages had the center of symmetry, which is an integral attribute of the diffraction patterns in the Fraunhofer zone for all objects, including non-centrosymmetrical (see eg. [38]), however, the rotational symmetry was not perfect: when one rotates the image around the axis with order of $k_{\alpha}$ for angles that are multiples of $180^{\circ} / k_{\alpha}$, only the positions of the diffraction reflexes coincide, but their intensity could vary. Part (Fig. 2b) or full (Fig. 2c-d) attenuation of the reflex intensities along some rays (similar to the extinction of the reciprocal lattice nodes in $\mathrm{x}$-ray analysis [39]).
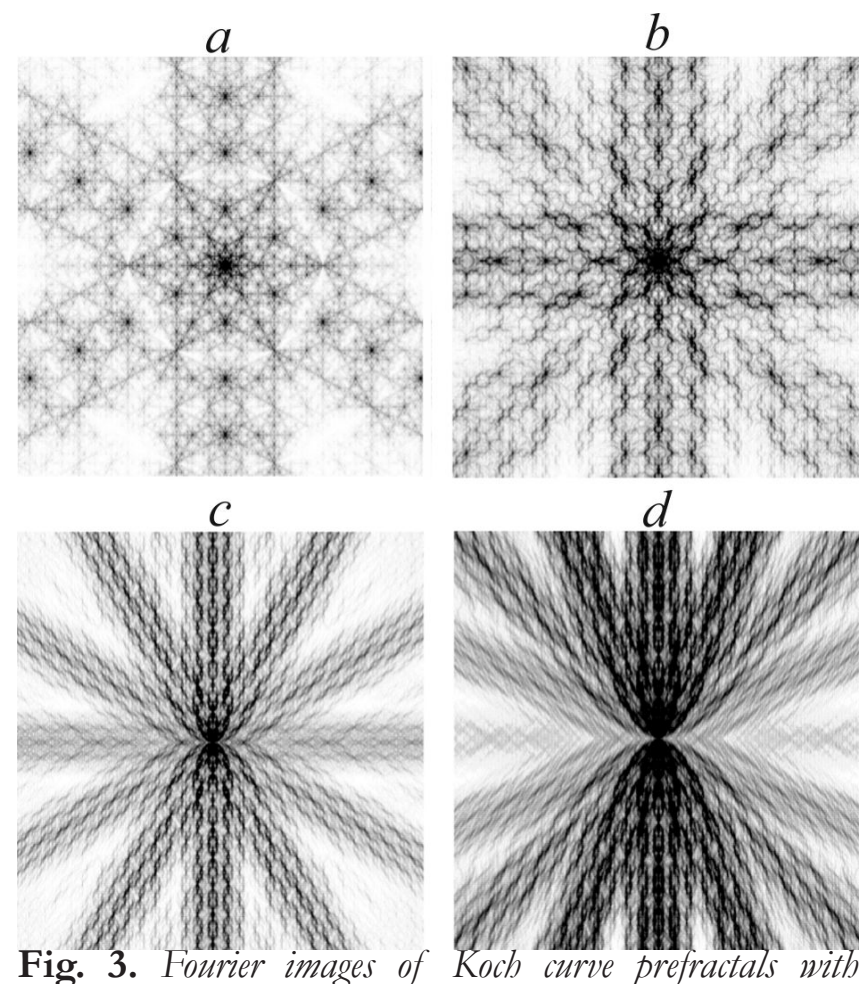

Fig. 3. Fourier images of Koch curve prefractals with quasisymmetry axis of 3-rd (a), 4-th (b), 6-th (c) and 8-th (d) order.
The cause of the observed features is that the objects under consideration, unlike crystals, are not a continuum of point objects (atoms), but a two-dimensional set of line segments with the same length $L / 3^{N_{g}}$, but with different orientation in space.

In this set for the integer values of $k_{\alpha}$ or $k_{y}$ one can stlect consisting of equally oriented line segments two-dimensional subsets, each of which contains a some number of partial one-dimensional diffraction gratings formed of located along the same line segments. In the general case these parallel latticies contain a different number of segments, and the degree of filling and the distance between adjacent segments, which determine the intensity and structure of the dis-tribution of diffraction reflects along the line, depend on the orientation of the lattice and the generation number of prefractal $N_{g}$.

The presence in each of the two-dimensional subsets of equally oriented segments together several spaced relative to each other parallel onedimensional lattices leads to a two-dimensional diffraction pattern with reflecs not only along the lattices, but on either side of them (the satellites in Fig. 2 and 4).

\section{SCHEME OF THE LATTICE AND FRACTAL PARTS OF THE FOURIER- IMAGES FORMATION}

The scheme of formation of the lattice part explains shown in Fig. 4 the example for Fourier image of 4-th generation prefractal with $\alpha=36^{\circ}$ having pentagonal quasisymmetry. The numbers $1-5$ in the image of prefractal

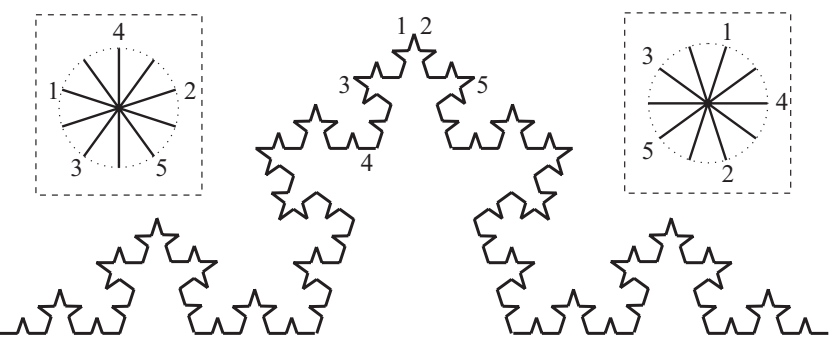

Fig. 4. The scheme of formation of the lattice part in Fourier image of 4-th generation Koch curve prefractal with $a=36^{\circ}$ having pentagonal quasisymmetry. 
marked 5 orientations of the segments; the inset on the right shows the possible directions of partial diffraction gratings, and the inset on left in the pull - direction of the rays (compare with Fig. 2). The number of partial lattices and the extent of their filling depends on the prefractal generation number. So, it follows from Fig. 4, that the number of partial sublattices in the subset with the direction of the segments of the 1-st type (see right inset) is 21 , and three of them contain 6 , 7 and 8 segments, two -4 four -3 , four -2 , and eight -1 .

When $\alpha=180^{\circ} / k_{\alpha}$ or $\gamma=180^{\circ} / k_{\gamma}$, where $k_{\alpha}$ and $k_{\gamma}$ are any integers, the number of possible orientations of the segments $N_{\mathrm{s}}$ in the prefractal is determined by the generation number of the latter $g$. When one initiates there are only three allowable orientations defined by the generator (Fig. 1a), each subsequent iteration adds two more.

In the case of even values of $k_{\alpha}$ or $k_{\gamma}$ on the step of the iteration, after which there are no segments with new orientations (i.e., when $N_{\mathrm{s}}=k_{\alpha}$ or $k_{\gamma}$ is added only one line segment, oriented perpendicular to the initiator. In subsequent iterations, the new directions of the segments are already not formed.

It was found that for even values of $k_{\alpha}$ there are $2 k_{\alpha}$ orientations of the line segments, and, accordingly, $2 k_{\alpha}$ rays in the lattice region of the Fourier image. For example, if $\alpha=22.5^{\circ}$ instead of 8 rays 16 is formed. We were not able to watch some of the rays due to a small population of the relevant partial sublattices even in prefractals of hightst accessible (for technical rea-sons) generations. For the same reasons, attempts failed to get quality Fourier image with decag-onal quasisymmetry.

Similar to the above-described properties are also inherent to Fourier images of the Koch snowflakes (with the initiator in the form of an equilateral triangle) shown in Fig. 5 for some prefractals of 6 -th generation with $\alpha=120^{\circ}-$ (a), $\alpha=22.5^{\circ}-(b), \alpha=30^{\circ}-(c)$, and $\alpha=12^{\circ}$

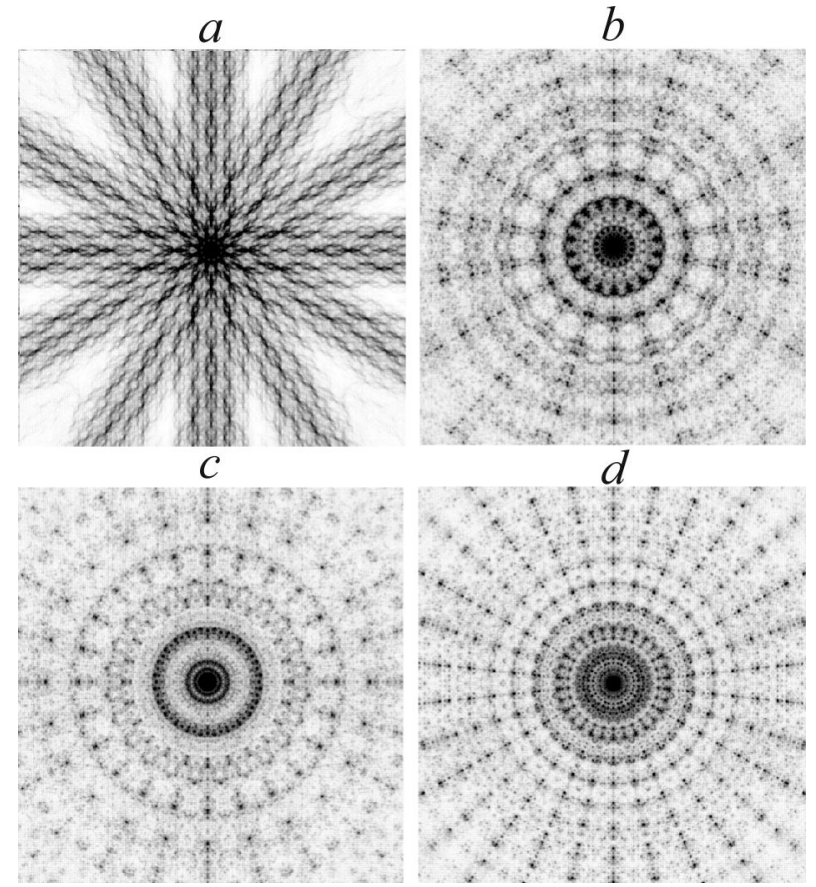

Fig. 5. Fourier images of Koch snowflakes prefractals with quasisymmetry axis of 6-th (a), 9-th (b), 12-th (c) and 15-th (d) order. - (d). Note that obtained in this case Fourier images are not a simple superposition of images from three sides of triangle initiator, as in the case of diffraction waves are summed taking into account phase difference between them.

As can be seen from Fig. 5, using triangle as initiator greatly enhances the filling of the partial sublattices and actually eliminates the difference in the intensity of diffraction spots in symmetry equivalent positions in all the rays of the lattice part of the Fourier image. In addition, in the Fourier images of the Koch snowflake one can more clearly trace the difference in structure of the central and peripheral parts. It is seen for example, that quasisymmetry of high orders manifests itself in the central part in a form of isolated spots, located on concentric circles.

Possessing self-similarity fractal part, the size of which enlarges with increasing angle (under rest equal conditions), is formed by the sum of the central diffraction maxima of all fractal ele-ments. At $L \rightarrow \infty$ and $N_{g} \rightarrow \infty$ the lattice contribution to the Fourier transform of the image disappears, as, in the first, the distance between the forming the lattices line 
segments tends to zero, and, in the second, the line segments transforms in the points, the diffraction pattern from which has radial symmetry.

\section{SUMMARY}

The above results convincingly show that the generalized triadic Koch prefractals of sufficiently high generations under certain conditions can have Fourier images with the axes of quasi-symmetry any order like parcuet mosaics [36] or quasicrystals [37]. Currently, there are data on realization of the mosaics with the quasisymmetry axis of the 5-th, 8-th, 10-th and 12-th order; in addition to these quasicrystals can have an axis of quasisymmetry of 7-th order [40, 41]. Amazing is the fact that unlike mosaics and quasicrystals Koch curves are generally deprived rotary axes of symmetry, and the Koch snowflakes have the only axis of symmetry of the 3 rd order.

However, it has been noticed long ago, that there exist some sibling connections among the fractals and parquet mosaics and also (discovered later) quasi-crystals; and in establishment of these connections, apparently, the decisive role played by self-similarity [40, 42]. And as the axioms are now perceived assertion that mosaics are two-dimensional analogs of quasicrystals, and to the last with any quasisymmetry can be put into correspondence associated fractal structures [43, 44]. Methods of fractal geometry are now used to describe models of quasi-crystalline structures, and tree-graph procedure - to originate them [44, 45].

From the point of view of abstract algebra aperiodic structures (quasi-crystals and mosaic), in contrast to describing by groups ideal crystals, are described by algebraic systems of "field" type, which may have a rotational symmetry and fractal ordering [46]. To describe geometric fractals, one can use the algorithms of so-called L-systems [47].

\section{REFERENCES}

1. Mandelbrot BB. The fractal geometry of nature. San Francisco, Freeman, 1983, 497 p.

2. Olemskoi AI, Flat AYa. UFN, 1993, 163(12):1-50 (in Russ.).

3. Gouyet J-F. Physics and fractal structures. Paris, Ecole Polytechnique, 1995, 234 p.

4. Peitgen HO, Jürgens H, Saupe D. Chaos and fractals. New frontiers of science. New York, Springer-Verlag, 2004, 864 p.

5. Levy-Vehel J, Lutton E. Fractals in Engineering. London, Springer-Verlag, 2005, 290 p.

6. Potapov AA. Fractaly v radiofizike $i$ radiolokatsii [Fractals in Radiophysisc and Radiolocation]. Moscow, Logos Publ., 2002, 847 p.

7. Fractals in Biology and Medicine: vol. IV (Mathematics and Biosciences in Interaction). Ed. by Losa GA,.Merlini D, Nonnenmacher TF, Weibel ER. Basel, Birkhaüser, 2005, 314 p.

8. Hudson RL, Mandelbrot BB. The (mis)Behavior of Markets: A Fractal View of Risk, Ruin, and Reward. New York, Basic Book, 2004, 328 p.

9. Almazov AA. Fractal'naya teoriya. Kak pomenyat' vaglyad na finansovye rynki [Fractal theory. How to change the view on the financial markets]. Moscow, Admiral Markets, 2009, 209 p.

10. Madden Ch. Fractals in Music: Introductory Mathematics for Musical Analysis. Salt Lake City, High Art Press, 1999, 224 p.

11. Bonch-Osmolovskaya TB. Vvedenie V literaturu formal'nyh ogranichenyi [An introduction to the literature of formal restrictions]. Samara, Bahrakh-M, 2009, 560 p.

12. Tarasenko VV. Fractal'naya semiotika. Slepye pyatna, peripetii i uznavaniya [Fractal semiotics. Blind spots, the vicissitudes and recognitions]. Moscow, Librokom, 2009, 232 p.

13. Isaeva VV, Kas'yanov NV. Vestnik DVO RAS, 2006, 5:119-127 (in Russ.).

14. Shlyk VA. Izv. Chelyabinskogo nauchnogo tsentra UrO RAS, 2004, 1(22):231-244 (in Russ.). 
15. Classification and Application of Fractals: New Research. Ed. by Mitchell EW, Murray SR. New York, October Nova, 2012, 347 p.

16. Alain C, Cloitre M. Phys. Rev. B, 1986, 33(5):3566-3569.

17. Uozumi J, Kimura H, Asakura T. J. Mod. Optics, 1990, 37(6):1011-1031.

18. Sakurada Y, Uozumi J, Asakura T. Opt. rev., 1994, 1(1):3-7.

19. Uozumi J, Kimura H, Asakura T. J. Mod. Optics, 1991, 38(7):1335-1347.

20. Sakurada Y., Uozumi J., Asakura T. J. Optics. A: Pure Appl.Optics, 1992, 1:29-40.

21. Chabassier G, Angéli B, Heliodore F, Le Mehauté AS. J. Optics. A: Pure Appl.Optics, 1992, 1:41-54.

22. Bo Hou, Gu Xu, Wen W, Wong GK L. Appl. Phys. Lett., 2004, 85(25):6125-6127.

23. Funamizu H., Uozumi J. J. Opt. Soc. of Amer.: Opt. Express, 2007, 15(12):7415-7422.

24. Horváth P, Śmid P, Vásková I, Hrabovský M. Optik, 2010, 121(2):206-213.

25. Arzamastseva GV, Evtikhov MG, Lisovsky FV, Mansvetova EG, Temiryazeva MP. ZhETP, 2008, 134(2):282-290 (in Russ.).

26. Arzamastseva GV, Evtikhov MG, Lisovsky FV, Mansvetova EG. ZhETP, 2011, 140(3):516-526 (in Russ.).

27. Peitgen HO, Richter PH. Beauty of Fractals: Images of Complex Dynamical Systems. SpringerVerlag, Berlin-Heidelberg, 1986, 199 p.

28. Arzamastseva GV, Evtikhov MG, Lisovsky FV, Mansvetova EG. Iqv. RAS, Ser. fir., 2010, 74(10):1430-1432 (in Russ.).

29. Arzamastseva GV, Evtikhov MG, Lisovsky FV,LukashenkoLI. Trudy XIXMę̧bdunarodnoi shkoly-seminara "Novye magnitnye materialy microelectroniki" [Proc. of the XX Intern. School-Seminar "New magnetic materials of microelectronics"], Moscow, 2004, p. 632634 (in Russ.).

30. Dikshtein IE, Kuznetsov DV, Lisovsky FV, Mansvetova EG. Trudy XVI Mę̧bdunarodnoi shkoly-seminara "Novye magnitnye materialy microelectroniki" [Proc. of the XX Intern.
School-Seminar "New magnetic materials of microelectronics"], p. II, Moscow, 1998:519 (in Russ.).

31. Lisovsky FV, Mansvetova EG, Lukashenko LI. Trudy XVI Mezhdunarodnoi shkoly-seminara "Novye magnitnye materialy microelectroniki" [Proc. of the XX Intern. School-Seminar "New magnetic materials of microelectronics"], Moscow, 2004, p. 838-840 (in Russ.).

32. Lisovsky FV., Lukashenko LI., Mansvetova EG. Pis'ma v ZhETP, 2004, 79(7):432-435 (in Russ.).

33. Arzamastseva GV., Evtikhov MG, Lisovsky FV, Mansvetova EG. Electromagnitnye volny $i$ electronnye sistemy, 2012, 17(12):29-32 (in Russ.).

34. Arzamastseva GV, Evtikhov MG, Lisovsky FV, Mansvetova EG. Electromagnitnye volny $i$ electronnye sistemy, 2012, 17(7):48-58 (in Russ.).

35. Arzamastseva GV, Evtikhov MG, Lisovsky FV, Mansvetova EG. Radioelektronika. Nanosistemy. Informatsionnye tekhnologii (RENSIT), 2012, 4(2):93-107 (in Russ.).

36. Penrose R. Eureka (Cambridge), 1978, 39:16-22.

37. Shechtman D, Blech LA, Gratias D, Cahn JW. Phys. Rev. Lett., 1984, 53(20):1951-1953.

38. Geht E. UFN, 1973, 111(2):355-364 (in Russ.).

39. Umansky YS, Skakov UA, Ivanov AM, Rastorguev LN. Kristallografiya, rentgenographiya $i$ elecnronnaya microscopiya [Crystallography, $\mathrm{X}$-ray and electron microscopy], Moscow, Metallurgiya, 1982, $632 \mathrm{p}$.

40. Suck J-B, Schreiber M, Haussler P. Quasicrystals: an introduction to structure, physical properties, and applications. Springer Verlag, Berlin-Heidelberg, 2010, 564 p.

41. Milman YuV, Efimov NA, Goncharova IV. Sborn. Nauchn. Trud. Inst. Probl. Materialoved. NAN Ukrainy, 2012, 18:3-14 (in Russ.).

42. Gardner M. Penrose Tiles to Trapdoor Ciphers. Freeman, New York, 1989, 416 p.

43. Lazarev AI, Domrachev GA. Kristallografiya, 1994, 39(5):811-844 (in Russ.). 
44. Yudin VV, Karygina UA. Kristallografiya, 2001, 46(6):1004-1008 (in Russ.).

45. Lazarev AI., Suhanov AU, Domrachev GA. Kristallografiya, 1996, 41(5):798-803 (in Russ.).

46. Domrachev GA, Lazarev AI. Fiz.tverdogo tela, 1999, 41(5):799 804 (in Russ.).

47. Lindenmayer A. J. Theor. Biology, 1968, 18:280315. 